EDITORIAL

\title{
B type natriuretic peptide testing: where are we now?
}

\section{R Cowie}

Heart 2004;90:725-726. doi: 10.1136/hrt.2003.014787

It is possible that plasma BNP measurement might be to heart failure what glycated haemoglobin measurement is to diabetes mellitus. Indeed, within 12-24 months, BNP testing might become a routine addition to the monitoring of patients with heart failure. In the meantime its main role is in helping to rule out heart failure in patients with new symptoms

Correspondence to: Professor Martin R Cowie, National Heart \& Lung Institute, Imperial College, London SW3 6LY, UK; m.cowie@imperial.ac.uk
T he plasma concentrations of B type natriuretic peptide (BNP), and the co-secreted but inactive aminoterminal proBNP, are raised in patients with heart failure. In general, the more severe the symptoms or the more severe the underlying cardiac abnormality, the higher the concentration. In principle, therefore, the measurement of plasma BNP concentrations may aid decision making in a variety of clinical settings. Assays are now straightforward, with turn around times of less than 20 minutes. As with any new technology, initial over enthusiasm has been followed by a more realistic assessment of its clinical value. ${ }^{1}$

BNP is a 32 amino acid peptide hormone secreted by the myocardium in response to stretch or strain. ${ }^{2}$ On secretion, proBNP, the storage form of BNP, is cleaved into the inactive $\mathrm{N}$ terminal proBNP and the endocrinologically active BNP. The active moiety causes vasodilatation, natriuresis, and diuresis, and as such helps to counteract the vasoconstriction and fluid retention triggered by many of the neurohormones that circulate at increased concentrations in patients with heart failure. In the USA, the Food and Drug Administration has licensed human recombinant BNP (Nesiritide) as a treatment for acute heart failure.

\section{DIAGNOSIS OF HEART FAILURE}

Several studies have examined the diagnostic utility of BNP in patients with heart failure. The highest value is found in studies of patients with new symptoms (such as breathlessness or fluid retention) who often have had no treatment. The overlap between the BNP concentration in such untreated new cases and those patients with new symptoms not caused by heart failure is small. Single centre studies, both in patients presenting to the emergency room ${ }^{3}$ or to rapid access clinics, ${ }^{4}$ report areas under the receiver operating characteristic (ROC) curves of above 0.90. Multicentre studies in similar populations report somewhat lower values, but still above $0.85 .^{56}$

The greatest value for BNP testing appears to be its ability to combine a very high negative predictive value with an acceptable positive predictive value. One can select a decision cut point below which heart failure as the cause of new symptoms is very unlikely (less than 5\%) and above that cut point $50-70 \%$ of patients will have heart failure confirmed on further assessment. The guidelines on heart failure from both the European Society of Cardiology, ${ }^{7}$ and the National Institute for Clinical Excellence (NICE), ${ }^{8}$ have recommended the use of BNP (or NTproBNP) testing as a "rule-out" test for patients with new symptoms.

A randomised comparison of a strategy of making NTproBNP results available to primary care physicians, in addition to the ECG, chest radiograph, and echocardiographic data, has reported a substantial increase in diagnostic accuracy for patients with new symptoms that might be caused by heart failure. ${ }^{9}$

The clinical situation is very different in patients with a "historical" diagnosis of heart failure-usually not confirmed by cardiac imaging as recommended by all recent national and international guidelines. The plasma concentration of BNP is likely to be lower in such patients, who are likely to have been treated with diuretics and angiotensin converting enzyme inhibitors. The overlap in terms of plasma BNP concentrations between abnormal and normal is greater. The diagnostic utility is therefore lower, with typical areas under the curve of between 0.5 to $0.8 .^{10}{ }^{11} \mathrm{BNP}$ is not an ideal "screening" test in such patients, and echocardiography (or other imaging) should be organised along with review of the previous evidence for heart failure.

\section{SCREENING FOR LEFT VENTRICULAR SYSTOLIC DYSFUNCTION}

Several population based studies have looked at the diagnostic value of BNP in identifying asymptomatic individuals within the general population who have left ventricular systolic dysfunction. The results vary depending on the study population, with highest values (as expected) in populations with a higher pre-test probability of disease, such as the elderly, and those with previous myocardial infarction. The North Glasgow study, based in the MONICA population, reported a negative predictive value of $97.5 \%$ and a positive predictive value of $32 \%$ in those over the age of $55 .{ }^{12}$ Another UK study

Abbreviations: ACS, acute coronary syndrome; BATTLESCARRED, BNP-assisted treatment to lessen serial cardiovascular readmissions and dealth; BNP, B type natriuretic peptide; MONICA, monitoring trends and determinants in cardiovascular disease; NICE, National Institute for Clinical Excellence; NTproBNP, N terminal proBNP; ROC, receiver operating characteristic; STARS, suivi du traitement dans l'insuffisance cardiaque systolique 
reported an area under the ROC curve of $0.85 .{ }^{13}$ Other studies have been less encouraging. ${ }^{1}$ In the UK, a screening programme using plasma BNP has not been adopted, although the evidence is under review.

\section{RISK STRATIFICATION}

Assessing prognosis in heart failure is not straightforward. Formal survival scores have been used, but chiefly to triage patients for consideration of heart transplantation. Functional severity, underlying cardiac dysfunction, systolic blood pressure, and renal function have consistently been shown to be associated with prognosis. BNP measurement might add to this. In one study of patients referred for transplantation in the era of "routine" $\beta$ blockade the only independent predictor of all cause mortality was the plasma NTproBNP concentration. ${ }^{14}$ Other small studies suggest that BNP measurement can be used to identify patients at low risk of hospital readmission. ${ }^{15}$ Currently, prognostication does not appear to be routine for patients with heart failure, particularly if they are elderly. The advent of expensive new technologies that may impact on prognosis-such as assist devices and combined atriobiventricular pacemaker/internal cardioverter defibrillators-may increase interest in this difficult area.

More surprisingly, perhaps, are the reports that plasma BNP concentration may provide prognostic information in patients with acute coronary syndrome (ACS). ${ }^{16} 17$ The mechanism of release of BNP in such patients is not clear, and appears to be independent of troponin release. Currently, international guidelines for the risk stratification of patients with ACS include troponin, but not BNP. This may change as evidence accumulates.

\section{TREATMENT MONITORING}

There has been much interest in the potential role of plasma BNP or NTproBNP in monitoring the clinical status of patients with heart failure. Conventional assessment is rather crude-querying symptoms, looking for signs of fluid retention, and examining trends in total body weight. It is possible that plasma BNP measurement might be to heart failure what glycated haemoglobin measurement is to diabetes mellitus. Two large randomised trials are underway (BATTLE-SCARRED, and STARS), following promising results from a very small single centre study from Christchurch, New Zealand. ${ }^{18}$ This study reported a clinically significant reduction in a composite end point of death, hospital admission, or episode of heart failure decompensation when patients were randomised to NTproBNP guided adjustment of treatment compared with conventional management $(p=0.02)$. As yet, the evidence is not robust, but if the value of BNP monitoring of heart failure is confirmed, this may have a huge impact on the request for such tests in routine care.

\section{PRACTICAL CONSIDERATIONS REGARDING ASSAY METHODS}

Several assay methods for BNP and NTproBNP are commercially available. ${ }^{1}$ The reference ranges, and decision cut points, depend on the assay method employed and the clinical question being addressed. There have been few headto-head comparisons of the different assay methods. Local experience is likely to fine tune decision cut points. The relative merits of point-of-care testing and central laboratory based assays have been discussed elsewhere. ${ }^{1}$ Often the key deciding factor will be local logistics and reimbursement issues. It is vital to ensure appropriate training of the individuals who will carry out the testing, and regular quality control assessment.

\section{CONCLUSIONS}

The measurement of plasma B type natriuretic peptide concentrations is now straightforward. The key factor in deciding whether such measurement should take place is whether the information provided adds value to current methods of arriving at a diagnosis or making treatment decisions, and at what cost. The evidence for its value in patients with new symptoms that might be caused by heart failure is robust, but even so the test should be used as part of a structured approach to the diagnosis of heart failure and should not stand alone-further investigation of "test positive" patients will be necessary. The other areas of use for BNP testing are, currently at least, of research interest only. Within 12-24 months, however, BNP testing may become a routine addition to the monitoring of patients with heart failure.

\section{REFERENCES}

1 Cowie MR, Jourdain P, Maisel A, et al. Clinical applications of B-type natriuretic peptide (BNP) testing. Eur Heart J 2003;24:1710-8.

2 Cowie MR, Mendez GF. BNP and congestive heart failure. Prog CV Disease 2002;44:293-321.

3 Dao Q, Khrishnaswamy $P$, Kazanegra R, et al. Utility of B-type natriuretic peptide in the diagnosis of congestive heart failure in an urgent-care setting. J Am Coll Cardiol 2001;37:379-85.

4 Cowie MR, Struthers AD, Wood DA, et al. Value of natriuretic peptides in assessment of patients with possible new heart failure in primary care. Lancet 1997;350:1349-51

5 Maisel AS, McCord J, Nowak RM, et al. Bedside B-type natriuretic peptide in the emergency diagnosis of heart failure with reduced or preserved ejection fraction. Results from the breathing not properly multinational study. J Am Coll Cardiol 2003;41:2010-7.

6 Zaphiriou A, Robb S, Murray-Thomas T, et al. Ruling out heart failure with BNP or NTproBNP: does it work in practice? Results of the UK natriuretic peptide study [abstract]. Heart 2003;89(suppl I):A49

7 The Task Force for the Diagnosis and Treatment of Chronic Heart Failure, European Society of Cardiology. Guidelines for the diagnosis and treatment of chronic heart failure. Eur Heart J 2001;22:1527-60.

8 National Institute for Clinical Excellence. Chronic heart failure: management of chronic heart failure in adults in primary and secondary care. London: NICE, 2003.

9 Wright SP, Doughty RN, Pearl A, et al. Plasma amino-terminal pro-brain natriuretic peptide and accuracy of heart-failure diagnosis in primary care: a randomized, controlled trial. J Am Coll Cardiol 2003;42:1793-800.

10 Hobbs FD, Davis RC, Roalfe AK, et al. Reliability of $\mathrm{N}$-terminal probrain natriuretic peptide in diagnosis of heart failure: cohort study in representative and high risk community populations. BMJ 2002;324:1498-502.

11 Vasan RS, Benjamin EJ, Larson MG, et al. Plasma natriuretic peptides for community screening for left ventricular hypertrophy and systolic dysfunction: the Framingham heart study. JAMA 2002;288:1252-9.

12 McDonagh T, Robb SD, Murdoch DR, et al. Biochemical detection of leftventricular systolic dysfunction. Lancet 1998;351:9-13.

13 Smith H, Pickering RM, Struthers $A D$, et al. Biochemical diagnosis of ventricular dysfunction in elderly patients in general practice: observational study. BMJ 2000;320:906-8

14 Gardner RS, Ozalp F, Murday AJ, et al. N-terminal pro-brain natriuretic peptide. A new gold standard in predicting mortality in patients with advanced heart failure. Eur Heart J 2003;24:1735-43.

15 Cheng V, Kazanagra R, Garcia A, et al. A rapid bedside test for B-type natriuretic peptide predicts treatment outcomes in patients admitted for decompensated heart failure: a pilot study. J Am Coll Cardiol 200;37:386-91.

16 de Lemos JA, Morrow DA, Bentley JH, et al. The prognostic value of B-type natriuretic peptide in patients with acute coronary syndromes. N Engl J Med 2001;345:1014-21.

17 Omland T, Persson A, Ng L, et al. N-terminal pro-B-type natriuretic peptide and long-term mortality in acute coronary syndromes. Circulation 2002; 106:2913-8.

18 Troughton RW, Frampton CM, Yandle TG, et al. Treatment of heart failure guided by plasma aminoterminal brain natriuretic peptide (N-BNP) concentrations. Lancet 2000;355:1126-30. 